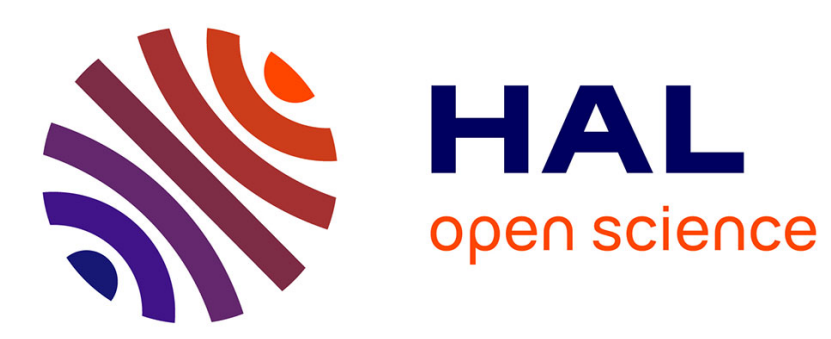

\title{
Disjoncteur ultra-rapide à couteaux : performances expérimentales et simulation théorique de son fonctionnement
}

\author{
P. Caupers, F. Rioux-Damidau
}

\section{To cite this version:}

P. Caupers, F. Rioux-Damidau. Disjoncteur ultra-rapide à couteaux : performances expérimentales et simulation théorique de son fonctionnement. Revue de Physique Appliquée, 1982, 17 (5), pp.337-347. 10.1051/rphysap:01982001705033700 . jpa-00245003

\section{HAL Id: jpa-00245003 https://hal.science/jpa-00245003}

Submitted on 1 Jan 1982

HAL is a multi-disciplinary open access archive for the deposit and dissemination of scientific research documents, whether they are published or not. The documents may come from teaching and research institutions in France or abroad, or from public or private research centers.
L'archive ouverte pluridisciplinaire $\mathbf{H A L}$, est destinée au dépôt et à la diffusion de documents scientifiques de niveau recherche, publiés ou non, émanant des établissements d'enseignement et de recherche français ou étrangers, des laboratoires publics ou privés. 
Classification

Physics abstracts

$85.20 \mathrm{Vq}-84.70+\mathrm{p}-06.70 \mathrm{Kv}$

\title{
Disjoncteur ultra-rapide à couteaux : performances expérimentales et simulation théorique de son fonctionnement
}

\author{
P. Caupers et F. Rioux-Damidau \\ Laboratoire d'Electrotechnique $\left(^{*}\right)$ des Universités Paris VI et XI, bâtiment 214, 91405 Orsay, France
}

(Reçu le 18 novembre 1981, révisé le 22 janvier 1982, accepté le 26 janvier 1982)

\begin{abstract}
Résumé. - Le disjoncteur élémentaire présenté permet de couper des courants élevés $(70 \mathrm{kA})$ en des temps très courts $(\sim 6 \mu \mathrm{s})$. Son ouverture est assurée par un système à pression magnétique directe, très efficace au point de vue énergétique. Les résultats expérimentaux sont présentés ainsi que la modélisation théorique du fonctionnement qui permet d'expliquer la courbe de réisolement intrinsèque.
\end{abstract}

\begin{abstract}
The hereafter presented elementary circuit-breaker can open high currents $(70 \mathrm{kA})$ in very short times $(\sim 6 \mu \mathrm{s})$. Its opening is ensured by a direct magnetic pressure system having high energetic efficiency. Firstly the experimental results are given. Next we show its theoretical simulation, which allows us to understand the switch intrinsic reinsulation properties.
\end{abstract}

Introduction. - La réalisation de sources d'énergie impulsionnelles très puissantes et peu onéreuses, capables de remplacer les bancs de condensateurs et même de dépasser largement leurs possibilités, passe maintenant par la mise au point de disjoncteurs à temps d'ouverture rapide $(\sim 10 \mu \mathrm{s})$ et à pouvoir de réisolement élevé. En effet, ils représentent l'élément final des systèmes de stockage inductif. Ils sont alors placés aux bornes d'une bobine magnétique qui est alimentée très simplement à l'aide d'une génératrice asynchrone sans fer [1], qui extrait l'énergie cinétique d'un volant en rotation.

Ces systèmes de coupure doivent être capables de ne pas trop s'échauffer pendant la durée de l'alimentation de la bobine et aussi d'absorber une quantité d'énergie importante lors de leur fonctionnement. C'est pourquoi les disjoncteurs rapides $[2,3]$ sont composés de deux éléments en parallèle, un " ouvreur " que le courant traverse dès le départ et qui subit ensuite une coupure mécanique et un "absorbeur", généralement une simple feuille de cuivre dont l'explosion génère la pointe de tension cherchée. L'un des impératifs les plus difficiles à satisfaire est d'obtenir l'ouverture de l'« ouvreur " en mettant en jeu une énergie dite "de commande " faible tout en assurant un bon réisolement. Dans une précédente publication [4], nous avons présenté un système répondant au critère ci-dessus et fonctionnant à l'aide de pressions

(*) Equipe de Recherche Associée au C.N.R.S. No 838. magnétiques impulsionnelles importantes fournies par un courant auxiliaire. Depuis, un disjoncteur utilisant les mêmes conceptions de base mais dont la structure générale a été grandement améliorée nous a permis d'obtenir, en particulier, de meilleurs réisolements [5].

Nous avons maintenant établi un modèle théorique qui permet de simuler le fonctionnement global de ce disjoncteur, en particulier en ce qui concerne ses caractéristiques de réisolement. Nous précisons ici les résultats expérimentaux, dont une partie a été donnée en [5] et présentons la modélisation du système.

1. Expérimentation. - 1.1 STRUCTURE DU DISJONCTEUR. - Le principe de base de l'ouvreur utilise comme en [4] la pression magnétique exercée entre deux conducteurs, parcourus par des courants en sens inverses l'un de l'autre. Sa structure schématique est indiquée figure 1. Les deux conducteurs en aluminium, de section rectangulaire, sont au début très proches et seulement séparés par une mince couche d'isolant.

Au départ, lorsque seul le courant principal $I_{\mathrm{P}}$ circule, les conducteurs s'attirent et par conséquent ne bougent pas. A un moment donné, on envoie le courant de demande $I_{\mathrm{C}}$. Si son intensité est supérieure à celle de $I_{\mathrm{P}}$, les courants dans chacun des conducteurs seront de sens opposés (Fig. 1). Comme le fusible n'est pas encore connecté $\left(I_{\mathrm{F}}=0\right)$ noús avons $I_{\mathrm{P}}=I_{0}$ et donc une force de répulsion, proportion- 


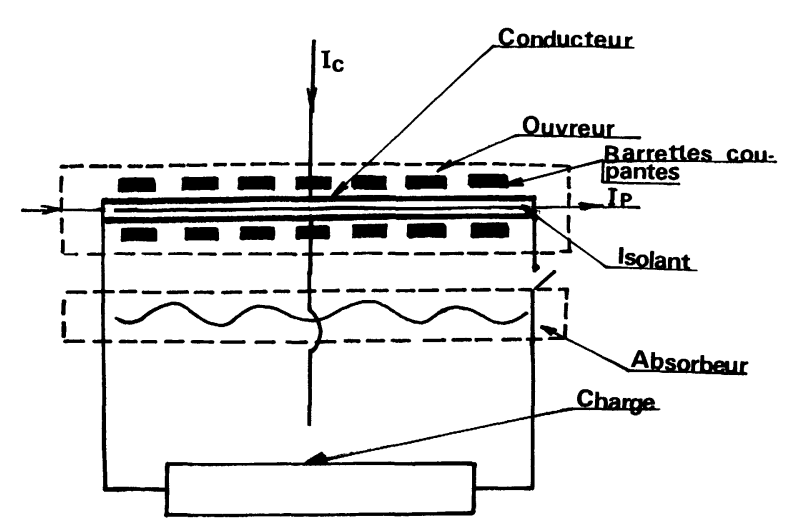

a)

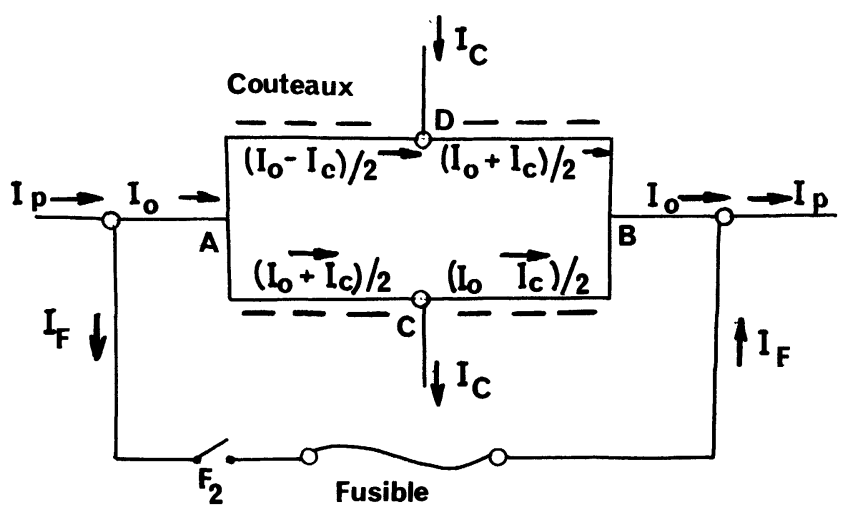

b)

Fig. 1. - Schéma du disjoncteur : (a) Structure. (b) Répartition des courants.

[Proposed switch principle : $(a)$ Structure. $(b)$ Currents distribution.]

nelle à $\frac{1}{4}\left(I_{\mathrm{C}}^{2}-I_{\mathrm{P}}^{2}\right)$. Celle-ci oblige les conducteurs en aluminium à aller se couper par cisaillement sur des barrettes coupantes qui sont placées de part et d'autre, perpendiculairement aux conducteurs.

A l'instant de coupure, le courant ne s'annule pas instantanément : des " arcs de rupture » apparaissent, assurant le transfert du courant vers un fusible, que l'on connecte à cet instant en parallèle. L'ouverture finale du circuit a lieu lors de l'explosion du fusible à condition que l'ouvreur tienne la surtension générée. Il est donc essentiel qu'après l'annulation du courant dans l'ouvreur, le milieu où viennent de se produire les arcs perde rapidement sa conductivité résiduelle. C'est pourquoi l'ensemble est placé dans une enceinte contenant un gaz isolant (cf. figure 4 de [4]). Nous avons ici utilisé de l'azote sous pression ce qui nous posait le minimum de problèmes expérimentaux tout en assurant un bon isolement. Maintenant que toutes les autres questions ont été résolues, il serait intéressant d'étudier le fonctionnement avec de l'hexafluore de soufre [6].

En ce qui concerne le bilan énergétique global, bien que l'intensité de $I_{\mathrm{C}}$ soit nécessairement plus grande que celle de $I_{\mathrm{P}}$, il est important de remarquer que l'énergie de commande peut être faible compara- tivement à l'énergie principale : il suffit pour cela que la condition $L_{\mathrm{C}} I_{\mathrm{C}}^{2} \ll L_{\mathrm{P}} I_{\mathrm{C}}^{2}$ soit satisfaite.

Les dimensions du système testé étaient les suivantes :

- conducteurs en aluminium : longueur $200 \mathrm{~mm}$ (connexions comprises), largeur $4 \mathrm{~mm}$, épaisseur $1 \mathrm{~mm}$;

- couteaux en acier : longueur $10 \mathrm{~mm}$, largeur $3 \mathrm{~mm}$, épaisseur $e_{\mathrm{c}}=1 \mathrm{~mm}$.

Les couteaux sont, au départ, contre les conducteurs, sauf ceux dits de réisolement (cf. $\S 2.3$ ). Les dimensions transversales du conducteur satisfont les conditions de rupture et d'échauffement établies en [4].

Les courants $I_{\mathrm{P}}$ et $I_{\mathrm{C}}$ sont fournis par deux bancs de condensateurs. Pour le courant principal, le quart de période est de $150 \mu \mathrm{s}$, son amplitude peut atteindre $70 \mathrm{kA}$; celle du courant de commande a toujours été comprise entre 160 et $185 \mathrm{kA}$, la durée totale du passage de courant étant de 25 à $30 \mu$ s (pour $I_{\mathrm{c}}<150 \mathrm{kA}$, les conducteurs ne sont pas coupés). Une évolution typique de ces courants est donnée figure 2 .

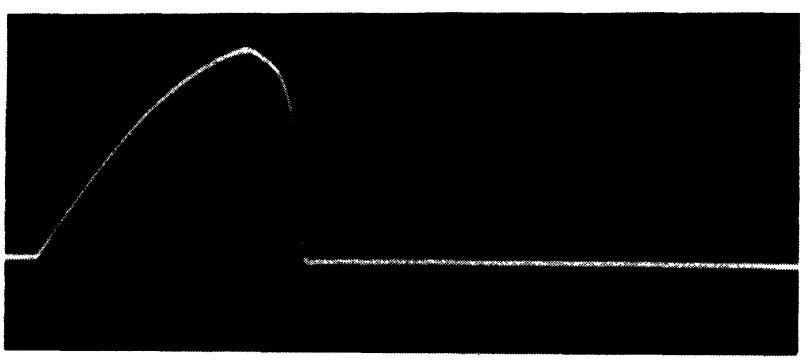

Fig. $2 a$. - Le courant principal $I_{\mathrm{P}}$. Le balayage vertical est de $16,6 \mathrm{kA} /$ division et le balayage horizontal de $50 \mu \mathrm{s} /$ division.

[The main current $I_{\mathrm{p}}$. Vertical scale is $16.6 \mathrm{kA} /$ division and horizontal scale $50 \mu \mathrm{s} /$ division.]

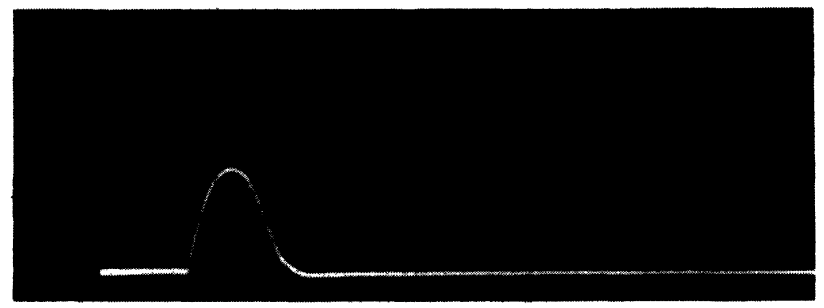

b)

Fig. 2b. - Le courant de commande $I_{\mathrm{C}}$. Les échelles sont de $22,3 \mathrm{kA}$ /division et $20 \mu \mathrm{s} /$ division. Le balayage dans le temps commence $88 \mu$ s après le démarrage de $I_{\mathrm{p}}$.

[The command current $I_{\mathrm{C}}$. Scales are $22.3 \mathrm{kA} /$ division and $20 \mu \mathrm{s} /$ division. Sweep trace is triggered $88 \mu \mathrm{s}$ after $I_{\mathrm{P}}$ begins to flow.]

1.2 TeNSIONS D'ARC ET TRANSFERT SUR LE FUSIBLE. - Lors de la rupture des conducteurs en aluminium sur les couteaux il apparaît une tension $V_{\mathrm{AB}}$ aux bornes principales de l'ouvreur qui est la différence 
entre les tensions $V_{\mathrm{AC}}$ et $V_{\mathrm{BC}}$ puisque les sens des courants dans les tronçons $\mathrm{AC}$ et $\mathrm{BC}$ sont opposés. Typiquement, au moment de la rupture, $75 \mathrm{kA}$ circulent dans $\mathrm{AC}$ et $30 \mathrm{kA}$ dans $\mathrm{BC}$ pour une amplitude de $I_{\mathrm{P}}$ voisine de $40 \mathrm{kA}$; les tensions correspondantes sont de l'ordre de $2500 \mathrm{~V}$ pour $V_{\mathrm{AC}}, 1500 \mathrm{~V}$ pour $V_{\mathrm{BO}}$ (si l'on place 4 couteaux sur chaque tronçon) et donc $1000 \mathrm{~V}$ pour $V_{\mathrm{AB}}$. Nous avons vérifié que $V_{\mathrm{AB}}$ et $V_{\mathrm{BC}}$ sont proportionnelles au nombre de couteaux puisqu'elles sont obtenues par addition des tensions des arcs générés par la rupture du conducteur sur chaque couteau ( 2 arcs par couteau).

La tension d'un arc dépend du courant $I$ qui le traverse et de sa dérivée temporelle $\mathrm{d} I / \mathrm{d} t$. Sur la figure 3 nous avons tracé la variation de la conductance $g=I / U$ d'un seul arc, ainsi que celle de la fonction $F=-\frac{\mathrm{d}}{\mathrm{d} t}(\log g)$, d'après les mesures de la

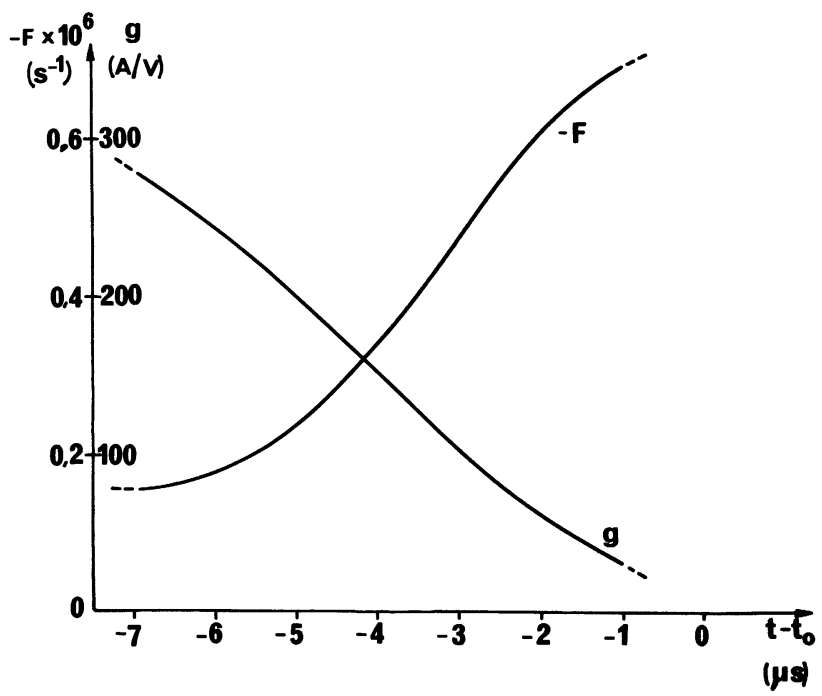

Fig. 3. - Variation temporelle lors du transfert du courant sur le fusible de la conductance $g$ d'un arc et de

$$
F=-\frac{\mathrm{d}}{\mathrm{d} t}(\log g)
$$

[Variation with time of both the conductance $g$ of an arc and of $F=-\frac{\mathrm{d}}{\mathrm{d} t}(\log g)$, during the current transfer onto the fuse.]

tension du tronçon $V_{\mathrm{AC}}$ et du courant lors d'une manipulation. $F$ est, en gros, liée à la constante de temps de l'arc par [7] :

$$
F=-\frac{1}{\tau}
$$

On constate que $\tau$ vaut environ 4 à $5 \mu$ s au début du transfert de courant puis diminue et tend vers $1 \mu$ s au fur et à mesure que l'on s'approche de l'annulation du courant. Notons que $F$ varie très peu d'un essai à l'autre.

Pour ce qui est du transfert du courant de l'ouvreur vers le fusible, il dépend de plusieurs paramètres, notamment de la tension $V_{\mathrm{AB}}$, mais aussi de la résistance $R_{\mathrm{F}}$ et de l'inductance $L_{\mathrm{F}}$ du circuit du fusible. Dans le montage expérimental utilisé, $L_{\mathrm{F}}$ valait environ $85 \mathrm{nH}$. Quant à $R_{\mathrm{F}}$ sa valeur à froid dépend évidemment des dimensions du fusible, mais en pratique, a toujours été comprise entre 5 et $15 \mathrm{~m} \Omega$. Les temps de transfert mesurés ont été compris entre 8 et $14 \mu \mathrm{s}$ : cette dispersion est essentiellement due à celle de $V_{\mathrm{AB}}$ qui dépend en particulier de l'instant de connexion du fusible, lequel dans le cas optimal, doit juste précéder l'instant de rupture des conducteurs.

\subsection{RÉISOLEMENT INTRINSÈQUE DE L'OUVREUR. -} La tension transitoire maximale $V_{R}$ supportable par l'ouvreur, que nous appellerons par la suite tension de reclaquage, est l'un des principaux critères de qualité du disjoncteur. En effet, celui-ci est destiné à effectuer un transfert d'énergie d'une bobine de stockage vers une charge en général inductive, transfert qui pourra être d'autant plus rapide que la tension $V_{F}$ générée par l'explosion du fusible sera plus grande. Cette tension $V_{\mathrm{F}}$ étant aussi appliquée aux bornes de l'ouvreur, elle doit donc vérifier $V_{F}<V_{R}$.

$V_{\mathrm{R}}$ dépend essentiellement de l'intervalle de temps $t_{\mathrm{R}}$ qui s'est écoulé depuis l'annulation du courant dans l'ouvreur (fin du transfert vers le fusible). Pour tracer les courbes de réisolement $V_{\mathrm{R}}\left(t_{\mathrm{R}}\right)$, nous avons fait différentes expériences en modifiant les dimensions du fusible ce qui changeait l'instant de la pointe de tension et nous notions à chaque fois $t_{\mathrm{R}}$ et $V_{\mathrm{R}}$ au moment du reclaquage, s'il avait lieu. Nous avons tout d'abord vérifié que la tension de reclaquage était proportionnelle au nombre de couteaux; les courbes de réisolement ont alors toujours été spécifiées par rapport à un seul couteau.

Sur la figure 4, nous avons tracé trois courbes de réisolement correspondant à des pressions d'azote dans la chambre de coupure et des courants de commande maximaux différents : 25 bars, $165 \mathrm{kA}$; 50 bars, $165 \mathrm{kA}$; 50 bars, $185 \mathrm{kA}$. Le courant principal valait $40 \mathrm{kA}$. Nous constatons que le passage de la pression de 25 à 50 bars se traduit par une tension de reclaquage plus de 2 fois supérieure et que l'augmentation du courant de commande améliore aussi grandement le réisolement. Dans ce dernier cas, cela est dû à une force de répulsion supérieure des conducteurs qui assure un balayage plus efficace des arcs et des morceaux cassés de conducteur. Malheureusement, cette amélioration du réisolement se paie par une plus grande détérioration des couteaux, qui ne dure plus qu'environ dix expériences au lieu de trente.

Une autre constatation de l'étude que nous avons menée concerne la non-influence sur le réisolement de l'amplitude du courant principal, tout au moins dans la gamme étudiée ( $20 \mathrm{kA}$ à $70 \mathrm{kA})$. Ceci est assez logique puisque les arcs dans l'ouvreur dépendent essentiellement du courant de commande qui est nettement supérieur au courant principal. 


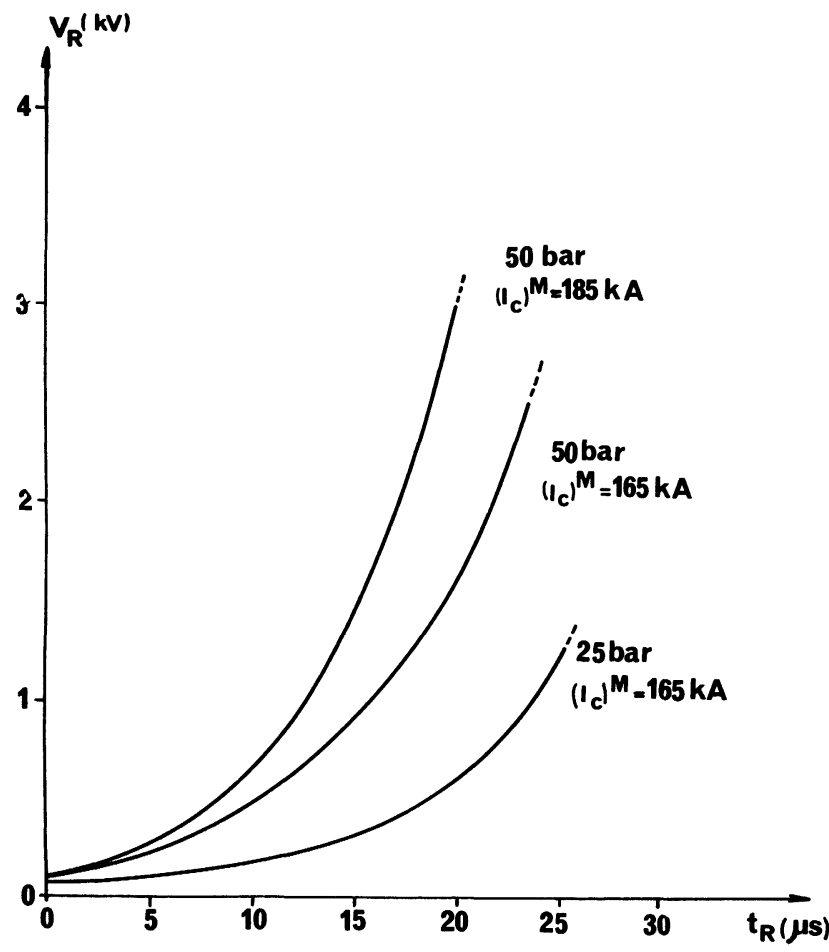

Fig. 4. - Courbe de réisolement intrinsèque (par couteau de rupture).

[The intrinsic reinsulation per cutting knife.]

1.4 AMÉlioration APPORTÉE PAR LES COUTEAUX DE RÉISOLEMENT. - Certains couteaux peuvent être décalés d'une distance $h$ par rapport à ceux qui sont placés, dés le départ, contre les conducteurs $(h=0)$. Ceci permet d'améliorer le réisolement, car si $h$ est judicieusement choisi, le conducteur se coupe sur les couteaux décalés alors que le courant vient de s'annuler, donc sans générer d'arcs. La figure 5 donne les courbes de réisolement (pour une pression de 50 bars et un courant de commande de $165 \mathrm{kA}$ ) pour diverses valeurs de $h$ : on constate que la valeur optimale de $h$ est $3 \mathrm{~mm}$ ce qui est normal puisque la vitesse des conducteurs est de l'ordre de $300 \mathrm{~m} / \mathrm{s}$ pendant le transfert du courant sur le fusible et que ce transfert dure environ $10 \mu \mathrm{s}$. Le gain obtenu est très appréciable, plus d'un facteur 3 par rapport aux couteaux non décalés.

1.5 BILAN ÉNERGÉTIQUE. - En mesurant la tension aux bornes de la commande ainsi que le courant $I_{C}$ nous avons pu déterminer la valeur de l'énergie de commande. Elle est de l'ordre de $2 \mathrm{~kJ}$ pour $I_{\mathrm{C}}=160 \mathrm{kA}$.

L'énergie absorbée par le disjoncteur vaut, en ordre de grandeur :

$$
W_{\mathrm{D}} \simeq V_{\mathrm{F}} I_{\mathrm{F}} t_{\mathrm{F}}
$$

où $V_{\mathrm{F}}, I_{\mathrm{F}}$ et $t_{\mathrm{F}}$ sont la tension générée par le fusible et donc supportée l'ouvreur, le courant dans le fusible qui est de l'ordre de $I_{\mathrm{P}}(<70 \mathrm{kA})$ et le temps d'ouverture du circuit, c'est-à-dire le temps d'explosion du fusible. Si l'on ajuste les dimensions du fusible pour

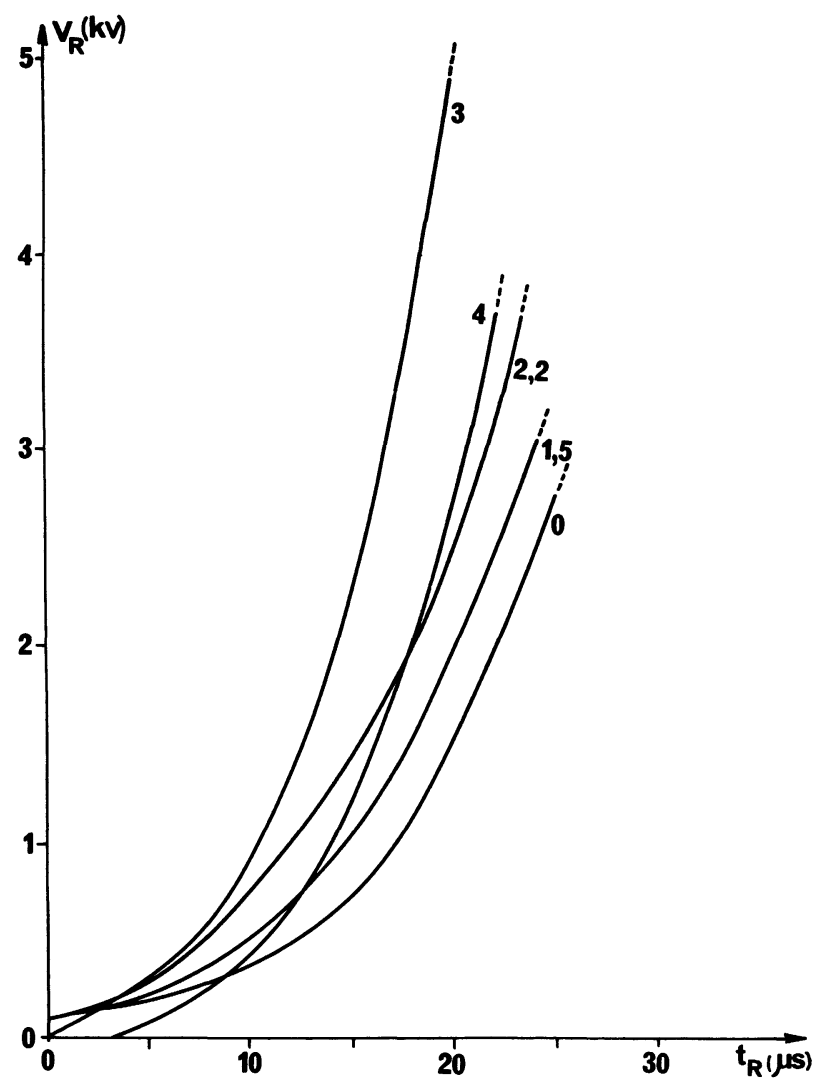

Fig. 5. - Courbe de réisolement par couteau de réisolement : les nombres indiqués près des courbes représentent le décalage $h$ en $\mathrm{mm}$.

[The switch reinsulation per reinsulating knife : the numbers near the curves are equal to the displacement $h$ in $\mathrm{mm}$.]

qu'il explose $20 \mu$ s après la fin du transfert du courant, lequel dure environ $10 \mu \mathrm{s}$, on a $t_{\mathrm{F}} \simeq 6 \mu \mathrm{s}$ et, pour $t_{\mathrm{R}}=20 \mu \mathrm{s}$, la tension admissible par le disjoncteur est de $1,5 \mathrm{kV}$ par couteau de rupture et $4,9 \mathrm{kV}$ par couteau de réisolement.

Dans notre montage où l'on met 14 couteaux par conducteur, par exemple 7 de rupture et 7 de réisolement, $19 \mathrm{~kJ}$ pourraient être absorbés ce qui conduit à une dépense énergétique de l'ordre de $10 \%$. Remarquons aussi que la quantité de matière détruite est très faible $\left(1 \mathrm{~cm}^{3}\right.$ pour $12 \mathrm{~kJ}$ absorbés), à peine supérieure à celle nécessaire pour une explosion de feuille. Ces pertes en énergie de commande et en matériau conducteur pourraient être encore diminuées si le nombre de couteaux de réisolement devenait prépondérant devant le nombre de couteaux de rupture, et/ou si les conducteurs étaient nettement plus longs de façon à ce que l'influence des connexions soit négligeable. Ce type de commande possède ainsi intrinsèquement une énergétique particulièrement favorable.

2. Modélisation du fonctionnement du disjoncteur. Nous avons modélisé les phases successives du fonctionnement du disjoncteur :

- passage du courant principal seul, 
- décharge de la commande, mise en vitesse et rupture des conducteurs,

- formation des arcs de rupture, transfert du courant vers le fusible,

- réisolement de l'ouvreur et explosion du fusible.

Du point de vue électrique, les circuits extérieurs au disjoncteur peuvent être représentés par les schémas des figures 6 et 11 . Quant à l'ouvreur, sa représentation est différente suivant que l'on se place dans le

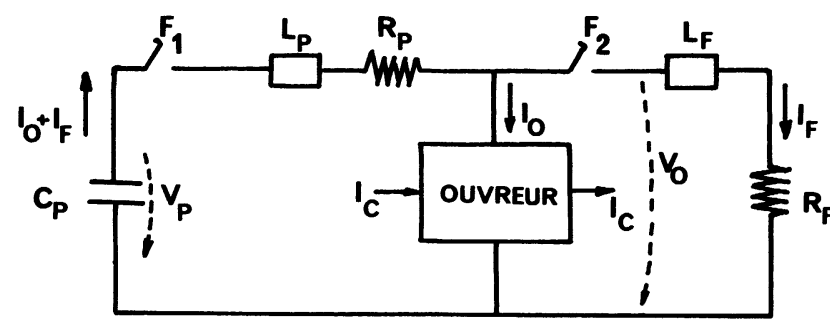

Fig. 6. - Schéma électrique du circuit du disjoncteur avec : $\mathrm{C}_{\mathrm{P}}$ : condensateur principal, $L_{\mathrm{P}}$ et $R_{\mathrm{P}}$ : inductance et résistance du circuit principal $L_{\mathrm{F}}$ et $R_{\mathrm{F}}$ : inductance et résistance du fusible, $F_{1}$ et $F_{2}$ : conjoncteurs.

[The circuit-breaker electrical diagram, with : $C_{\mathrm{P}}:$ Main capacitor, $L_{\mathrm{P}}$ and $R_{\mathrm{P}}$ : inductance and resistance of main circuit, $L_{\mathrm{F}}$ and $R_{\mathrm{F}}$ : inductance and resistance of the fuse, $F_{1}$ and $F_{2}:$ switches.]

circuit principal ou dans celui de commande. Le champ magnétique entre les conducteurs engendré par le courant principal est très faible et l'inductance de l'ouvreur vue du circuit principal est pratiquement indépendante de la position des conducteurs. Ce n'est pas du tout le cas si l'on considère la situation par rapport au courant de commande, puisque celui-ci engendre un flux important entre eux.

2.1 Modélisation De La PRemière PhaSe. - Pendant cette première phase, les conjoncteurs $F_{2}$ et $F_{C}$ restent en position ouverte, de sorte que l'ouvreur est traversé par tout le courant principal. Le courant $I$ obéit à :

$$
\frac{\mathrm{d} I_{0}}{\mathrm{~d} t}=\frac{1}{L_{\mathrm{P}}+L_{0}}\left[V_{\mathrm{P}}-I_{0}\left(R_{\mathrm{P}}+R_{0}\right)\right]
$$

où $V_{\mathrm{P}}$ représente la tension instantanée aux bornes du banc de condensateur principal, de capacité $C_{\mathrm{p}}$. Cette tension $V_{\mathrm{P}}$ obéit à

$$
\frac{\mathrm{d} V_{\mathrm{P}}}{\mathrm{d} t}=-\frac{1}{C_{\mathrm{P}}} I_{0}
$$

avec $V_{\mathrm{P}}=V_{\mathrm{o}}$ pour $t<0$.

Le courant de commande et celui dans le fusible sont nuls.

2.2 Modélisation De LA Deuxième PHASE. Pendant la deuxième phase de fonctionnement, le conjoncteur $\mathrm{F}_{\mathrm{C}}$ est fermé et l'impulsion de commande circule. Les conducteurs se repoussent jusqu'à se cisailler sur les couteaux de rupture. Le courant dans le fusible est toujours nul, puisque celui-ci n'est pas encore connecté.

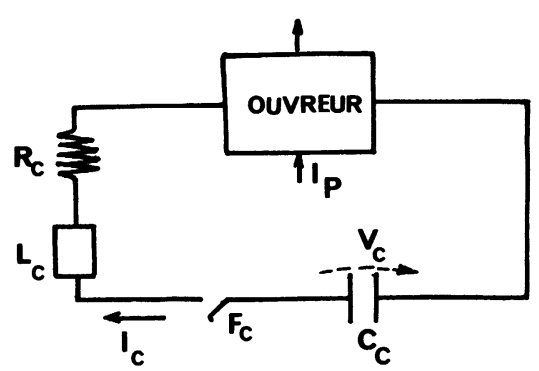

Fig. 7. - Schéma électrique du circuit de commande avec : $C_{\mathrm{C}}$ : condensateur de commande, $L_{\mathrm{C}}$ et $R_{\mathrm{C}}$ : inductance et résistance $\mathrm{F}_{\mathrm{C}}$ : conjoncteur.

[Electrical diagram of the command circuit with : $C_{\mathrm{c}}=$ capacitor, $L_{\mathrm{C}}$ and $R_{\mathrm{C}}$ : inductance and resistance, $\mathrm{F}_{\mathrm{C}}$ : switch.]

2.2.1 Equations électriques. - Par circulation dans les mailles principales et de commande, on peut déduire facilement les équations auxquelles obéissent les courants $I_{0}$ dans l'ouvreur et $I_{\mathrm{C}}$ de commande :

$\frac{\mathrm{d} I_{0}}{\mathrm{~d} t}=\frac{1}{L_{\mathrm{P}}+L_{0 \mathrm{P}}}\left[V_{\mathrm{P}}-I_{0}\left(R_{\mathrm{P}}+R_{0}\right)\right]$

$\frac{\mathrm{d} I_{\mathrm{C}}}{\mathrm{d} t}=\frac{1}{L_{\mathrm{C}}+L_{\mathrm{oc}}}\left[V_{\mathrm{C}}-I_{\mathrm{C}}\left(R_{\mathrm{C}}+R_{0}+\frac{\mathrm{d} L_{0 \mathrm{C}}}{\mathrm{d} t}\right)\right]$

où $L_{0 \mathrm{p}}$ et $L_{\mathrm{oC}}$ sont les inductances de l'ouvreur respectivement dans les circuits principal et de commande.

En ce qui concerne les tensions des bancs de condensateurs, elles sont définies par (3) et :

$$
\frac{\mathrm{d} V_{\mathrm{C}}}{\mathrm{d} t}=-\frac{1}{C_{\mathrm{C}}} I_{\mathrm{C}}
$$

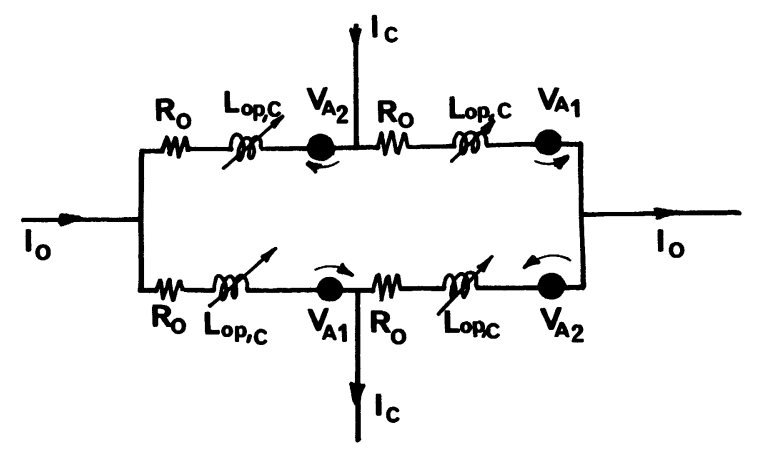

Fig. 8. - Schéma électrique de l'ouvreur. L'inductance est $L_{0 P}$ vue du circuit principal et $L_{0 \mathrm{C}}$ vue du circuit de commande. $V_{\mathrm{A} 1}$ et $V_{\mathrm{A} 2}$ sont nuls pendant les phases 1 et 2 .

[Electrical diagram of the " opener ". Its inductance is $L_{0 P}$ when seen from the main circuit and $L_{0 \mathrm{C}}$ when seen from the command circuit. $V_{\mathrm{A} 1}$ and $V_{\mathrm{A} 2}$ are zero during phases 1 and 2.] 
2.2.2 Loi de variation de l'inductance. - Pour obtenir $L_{0 C}$, on effectue le calcul intermédiaire de l'induction magnétique $B$ régnant entre les conducteurs, en remarquant que comme ceux-ci sont très longs comparés à leurs dimensions transversales, on peut traiter le problème en deux dimensions en négligeant les effets de bouts. Le calcul se fait analytiquement et est relativement lourd [8]. On trouve que :

$$
L_{\mathrm{oC}}=\frac{\mu_{0} D}{2 \pi d e}(Q(b)+Q(e)-Q(e+b))
$$

avec

$Q(x)=x d \log \left(1+\frac{x^{2}}{d^{2}}\right)+d^{2} \operatorname{arctg} \frac{x}{d}+x^{2} \operatorname{arctg} \frac{d}{x}$

où $D, 2 d$ et $e$ sont respectivement la longueur, la largeur et l'épaisseur d'un conducteur. $b$ est la distance variable en fonction du temps entre les conducteurs.

2.2.3 Force exercée sur les conducteurs. - Cette force est uniquement une force de répulsion entre les conducteurs puisqu'ils sont en face l'un de l'autre; il n'y a pas d'effort tangentiel. Le calcul en est assez fastidieux, quoique classique. On obtient [8], pour la force par unité de longueur du conducteur :

$F_{\mathrm{e}}=-\mu_{0} j_{\mathrm{a}} j_{\mathrm{b}}(2 Z(b+e)-Z(b+2 e)-Z(b))$

avec

$$
\begin{aligned}
Z(x)= & x d^{2} \log \left(x^{2}+4 d^{2}\right)-\frac{x^{3}}{12} \times \\
& \times \log \left(1+4 \frac{d^{2}}{x^{2}}\right)+\frac{4 d^{3}}{3} \operatorname{arctg} \frac{x}{2 d}+x^{2} d \times \\
& \times \operatorname{arctg} \frac{2 d}{x}
\end{aligned}
$$

$j_{\mathrm{a}}$ et $j_{\mathrm{b}}$ représentent les densités de courant uniformes circulant dans chaque conducteur.

2.2.4 Loi du mouvement. - Le travail des forces de pression magnétique communique une certaine énergie cinétique à chaque morceau de conducteur de masse $M$ situé entre deux couteaux. Mais une partie de l'énergie $\mathrm{d} W_{\mathrm{d}}$ est aussi dissipée par la déformation des conducteurs. La conservation de l'énergie impose toutefois que :

$$
F_{\mathrm{e}} \mathrm{d} x=M v \mathrm{~d} v+\mathrm{d} W_{\mathrm{d}}
$$

avec : $M=2 \bar{\omega} c d e(\omega$ étant la masse volumique de l'aluminium).

La rupture ayant lieu par cisaillement, l'énergie de déformation s'obtient à partir de la contrainte de cisaillement (cf. Fig. 9)

$$
\sigma_{\mathrm{c}}=F_{\mathrm{e}} / 4 d e .
$$

Cependant, la valeur de la contrainte provoquant la rupture ainsi que l'angle de déformation correspon-

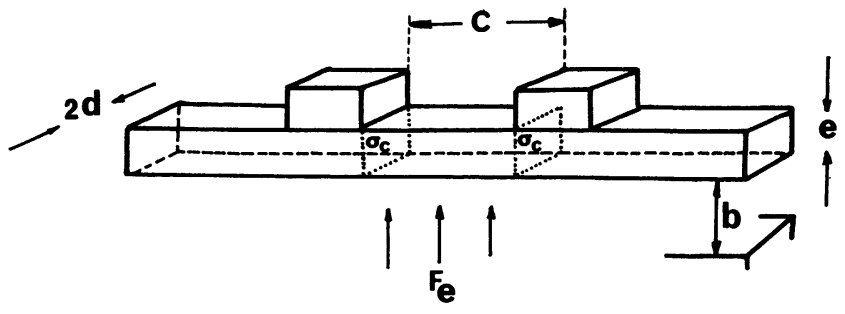

Fig. 9. - Dimensions des conducteurs. La force $F_{\mathrm{e}}$ engendre des contraintes de cisaillement $\sigma_{\mathrm{c}}$.

[The conductors' dimensions. The force $F_{\mathrm{e}}$ causes a shear stress $\sigma_{\mathrm{c}}$.]

dant dépendent fortement de la vitesse puisque nous ne sommes pas dans un essai statique. Nous sommes donc amenés à utiliser nos résultats expérimentaux : nous avons trouvé une contrainte à la rupture d'environ $16 \mathrm{daN} / \mathrm{mm}^{2}$, soit à peu près le double de celle prévue dans les tables pour des essais statiques. Quant à l'angle de déformation, nous prendrons aussi une valeur double de celle donnée dans les tables, ce qui est normal dans des essais dynamiques [9]; le diagramme de cisaillement dynamique de nos expériences est donc celui de la figure 10.

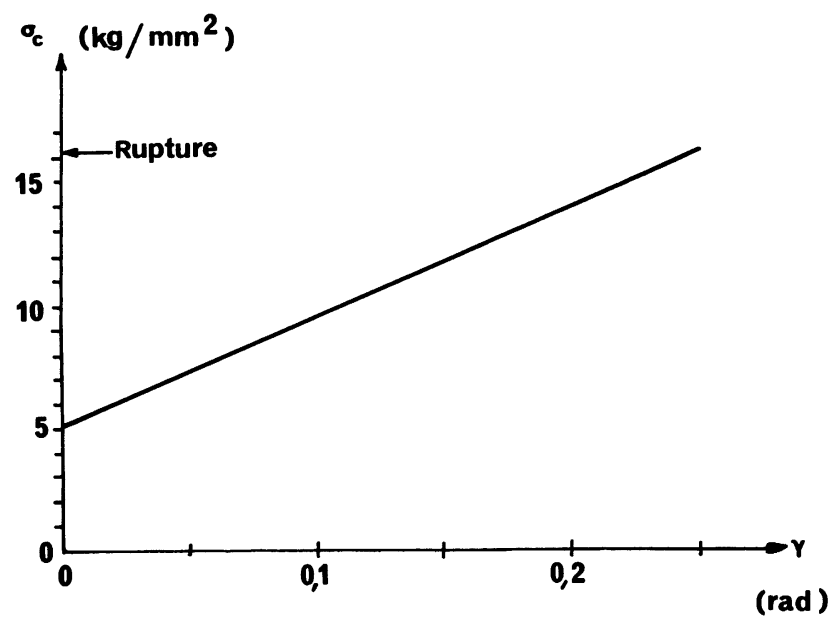

Fig. 10. - Diagramme de cisaillement dynamique adopté pour nos simulations.

[Dynamic shear diagram considered for our calculation.]

La valeur de l'énergie dissipée dans le processus de déformation est alors donnée par :

$$
W_{\mathrm{d}}=V_{\mathrm{d}} \int_{0}^{\gamma} \sigma_{\mathrm{c}} \mathrm{d} y
$$

où $V_{\mathrm{d}}$ est le volume déformé. Or pour chaque morceau de conducteur il y a deux zones de déformation, une à chaque bout. Par suite :

$$
V_{\mathrm{d}}=4 \text { lde }
$$

où $l$ est une longueur que nous prendrons égale à $0,4 \mathrm{~mm}$ d'après les constatations expérimentales. 
Finalement, on en déduit l'équation du mouvement des morceaux de conducteurs :

$$
2 \bar{\omega} d e \frac{\mathrm{d} v}{\mathrm{~d} t}=F_{\mathrm{e}}-1 \frac{d}{\mathrm{~d} t}\left(\int_{0}^{\gamma} F_{\mathrm{e}} \cdot \mathrm{d} \gamma\right)
$$

où $F_{\mathrm{e}}$ est donné par $(8)$; $\gamma$ se lit sur la figure 10 connaissant $\sigma_{\mathrm{c}}$ par (9).

2.2.5 Processus de calcul. - L'équation (10) cidessus a été résolue numériquement à l'aide d'une méthode de calcul pas-à-pas. La procédure cyclique de calcul est la suivante :

à (6);

- résoudre les équations électriques du système (2)

- calculer la force de répulsion $F_{\mathrm{e}}(8)$ et la contrainte de cisaillement $\sigma_{\mathrm{c}}$ (9) pour la position $x$ des conducteurs ;

- calculer l'énergie de déformation, ainsi que sa dérivée temporelle;

- résoudre l'équation du mouvement (10). On obtient la vitesse $v$, et par intégration la nouvelle position des conducteurs;

- à partir de cette nouvelle position, calculer l'inductance $L_{0 \mathrm{C}}$ de l'ouvreur (7);
- recommencer le cycle, jusqu'à ce que la contrainte de cisaillement soit suffisante pour assurer la rupture (dans notre cas $\sigma_{\mathrm{c}}=16 \mathrm{kgf} / \mathrm{mm}^{2}$ ) ;

- la rupture étant achevée, le calcul doit se poursuivre avec une énergie de déformation nulle. La fin de cette phase a lieu pour un déplacement égal à la somme des épaisseurs du conducteur et du couteau. Il est à remarquer que nous considérons que, pendant que le morceau se déplace entre les deux couteaux, il y a encore un bon contact métallique entre ceux-ci et le conducteur.

2.3 Modélisation DE LA TROISIÈMe PHASE. - La troisième phase de fonctionnement commence avec l'apparition des arcs (ouverture de l'ouvreur). Le courant $I_{0}$ se transfère progressivement vers le fusible, que l'on suppose connecté au moment de l'ouverture (cas optimal), par la fermeture du conjoncteur à fil $\mathrm{F}_{2}$ (Fig. 6).

2.3.1 Equations électriques. - Par circulation dans les mailles principale et de commande (Figs. 6, 7, 8), nous pouvons déduire les équations donnant $I_{0}, I_{\mathrm{F}}$ et $I_{\mathrm{C}}:$

$$
\begin{aligned}
& \frac{\mathrm{d} I_{0}}{\mathrm{~d} t}=\frac{L_{\mathrm{F}}}{L_{\mathrm{P}} L_{\mathrm{F}}+L_{\mathrm{P}} L_{\mathrm{OP}}+L_{\mathrm{F}} L_{\mathrm{OP}}}\left[V_{\mathrm{P}}-I_{0}\left(R_{\mathrm{P}}+R_{0}+R_{0} \frac{L_{\mathrm{P}}}{L_{\mathrm{F}}}\right)-\right.\left.-I_{\mathrm{F}}\left(R_{\mathrm{P}}-R_{\mathrm{F}} \frac{L_{\mathrm{P}}}{L_{\mathrm{F}}}\right)+\left(1+\frac{L_{\mathrm{P}}}{L_{\mathrm{F}}}\right)\left(V_{\mathrm{A} 2}-V_{\mathrm{A} 1}\right)\right] \\
& \frac{\mathrm{d} I_{\mathrm{F}}}{\mathrm{d} t}=\frac{1}{L_{\mathrm{P}}+L_{\mathrm{F}}}\left[V_{\mathrm{P}}-R_{\mathrm{P}} I_{0}-\left(R_{\mathrm{P}}+R_{\mathrm{F}}\right) I_{\mathrm{F}}-\frac{L_{\mathrm{P}} L_{\mathrm{F}}}{L_{\mathrm{P}} L_{\mathrm{F}}+L_{\mathrm{P}} L_{\mathrm{OP}}+L_{\mathrm{F}} L_{\mathrm{OP}}} \times\right. \\
&\left.\times\left[V_{\mathrm{P}}-I_{0}\left(R_{\mathrm{P}}+R_{0}+R_{\mathrm{O}} \frac{L_{\mathrm{P}}}{L_{\mathrm{F}}}\right)-I_{\mathrm{F}}\left(R_{\mathrm{P}}-R_{\mathrm{F}} \frac{L_{\mathrm{P}}}{L_{\mathrm{F}}}\right)+\left(1+\frac{L_{\mathrm{P}}}{L_{\mathrm{F}}}\right)\left(V_{\mathrm{A} 2}-V_{\mathrm{A} 1}\right)\right]\right] \\
& \frac{\mathrm{d} I_{\mathrm{C}}=\frac{1}{\mathrm{~d} t}+V_{\mathrm{C}}+L_{\mathrm{OC}}}{\left.L_{\mathrm{C}}-\left(R_{0}+R_{\mathrm{C}}+\frac{\mathrm{d} L_{0 \mathrm{C}}}{\mathrm{d} t}\right) I_{\mathrm{C}}-V_{\mathrm{A} 1}-V_{\mathrm{A} 2}\right] .}
\end{aligned}
$$

Dans ces équations, $V_{\mathrm{A} 1}$ et $V_{\mathrm{A} 2}$ représentent les chutes de tension dues aux arcs, respectivement pour la moitié de conducteur traversée par le courant $\frac{1}{2}\left(I_{\mathrm{C}}+I_{0}\right)$, et pour la moitié de conducteur traversée par $\frac{1}{2}\left(I_{\mathrm{C}}-I_{0}\right)$. Quant au courant principal $I_{\mathrm{P}}$ il vaut évidemment

$$
I_{\mathrm{P}}=I_{\mathrm{F}}+I_{0}
$$

La tension instantanée aux bornes du condensateur principal obéit, elle, à :

$$
\frac{\mathrm{d} V_{\mathbf{P}}}{\mathrm{d} t}=\frac{I_{\mathbf{P}}}{C_{\mathbf{P}}}
$$

tandis que celle du banc de condensateurs de la commande est encore donnée par (6).

Il nous reste encore à modéliser les arcs, afin de connaître les tensions $V_{\mathrm{A} 1}$ et $V_{2}$.
2.3.2 Les arcs dans le disjoncteur. - De nombreux modèles relativement complexes [10], [11], [12], [13] ont été proposés. Nous avons choisi ici d'adopter la théorie de l'arc proposée par Hochrainer et Grütz [14], qui met en jeu un minimum d'hypothèses ce qui a priori permet de l'appliquer à toutes sortes d'arcs dans les disjoncteurs. Elle est basée sur le fait que l'arc est un dipôle non linéaire, ayant une conductance instantanée $g=I / V$, et dans lequel une puissance $P=V I$ est produite. Cette puissance augmente la quantité d'énergie $Q$ contenue dans l'arc, tandis qu'au même instant une puissance $P_{\mathrm{a}}$ est dissipée vers l'environnement. L'on aura donc :

$$
\frac{\mathrm{d} Q}{\mathrm{~d} t}=P-P_{\mathrm{a}} \text {. }
$$

Plus l'énergie $Q$ emmagasinée dans l'arc est grande, 
plus la température de celui-ci est élevée, ce qui réagit sur sa conductance $g$. La puissance $P_{\mathrm{a}}$ dissipée dépend non seulement de la température, mais aussi du mécanisme de refroidissement. L'arc se présente donc, selon cette théorie comme une sorte de servomécanisme bouclé.

A partir de l'équation de conservation de l'énergie (16) Hochrainer déduit après quelques calculs l'équation donnant la variation de la conductance instantanée $g$ de l'arc

$$
\frac{\mathrm{d} g}{\mathrm{~d} t}=\frac{1}{\tau}(G-g)
$$

avec $G$ : conductance de l'état stationnaire que l'on obtiendrait si l'on maintenait le courant d'arc constant pendant un temps suffisamment long; $\tau$ : constante de temps de l'arc.

L'équation (17) permet, si l'on connaît les deux fonctions $G=G(I)$ et $\tau=\tau(I)$, de prévoir l'interaction entre un disjoncteur et son réseau extérieur.

Regardons tout d'abord la constante de temps. Nous savons que lors du transfert du courant vers le fusible, le courant qui traverse l'ouvreur diminue très rapidement. Il est donc clair qu'à chaque instant la conductance instantanée $g$ est notablement supérieure à la conductance stationnaire $G$ relative au même courant d'arc. Nous avons alors

$$
\frac{1}{\tau} \gtrsim-\frac{\mathrm{d}}{\mathrm{d} t}(\log g)
$$

et l'on peut obtenir l'ordre de grandeur de $\tau$ d'après la mesure expérimentale de la conductance $g$ (cf. Fig. 3).

Nous avons ainsi adopté la fonction $g(I)$ suivante :

$-\tau \simeq 1 \mu \mathrm{s}$ si $I \leqslant 1 \mathrm{kA}$,

$-\tau \simeq(0,96+0,04 I) \mu \mathrm{s}$ avec $I$ en kA, si $I>1 \mathrm{kA}$.

En ce qui concerne la conductance d'un arc stationnaire $G=V / I$, sa variation avec le courant dépend de l'ordre de grandeur de l'intensité. Pour les courants élevés ( $>1 \mathrm{kA}$ ), la caractéristique d'un arc brûlant dans un milieu fortement ionisé a une pente positive et l'on a [15] :

$$
V=m+n I
$$

soit

$$
G=\frac{m}{I}+n
$$

Il en est ainsi pendant la quasi-totalité du transfert de courant. Vers la fin, pour $I<1 \mathrm{kA}$, la caractéristique devient différente [14] :

soit

$$
V=a+b / I
$$

$$
G=(a I+b) / I^{2}
$$

$a$ et $b$ sont des paramètres dépendant de la nature des électrodes et du gaz dans lequel l'arc brûle. Pour des électrodes en aluminium et un milieu d'azote à
1 bar [17], $a \simeq 48 \mathrm{~V}, b \simeq 23 \mathrm{VA}$. Dans les équations (18) et (19) la longueur de l'arc n'intervient pas car ce sont les chutes de tension aux niveaux de la cathode et de l'anode qui sont prépondérantes.

La bibliographie ne donne pas les valeurs de $a, b, m$ et $n$ dans les conditions de nos expériences, aussi nous avons procédé par tentatives jusqu'à obtenir des simulations théoriques proches de la réalité ce qui nous conduit à prendre :

$$
\begin{gathered}
a=99 \mathrm{~V}, \quad b=100 \mathrm{VA}, \quad m=89 \mathrm{~V}, \\
n=0,011 \mathrm{~V} / \mathrm{A} .
\end{gathered}
$$

Nous avons pu ainsi exprimer les tensions d'arc $V_{\mathbf{A} 1}$ et $V_{\mathrm{A} 2}$ relatives aux moitiés de conducteur traversées par les courants $\frac{1}{2}\left(I_{C}+I_{0}\right)$ et $\frac{1}{2}\left(I_{C}-I_{0}\right)$ en tenant compte de ce que chaque couteau de rupture génère 2 arcs.

2.3.3 La résistance variable du fusible. - L'explosion de fusibles a déjà fait l'objet de nombreuses études approfondies [18] [19] [20]. Il s'agit néanmoins d'un sujet assez délicat, car les expériences montrent clairement que la variation de résistivité dépend de la vitesse de déposition de l'énergie Joule (phénomène d'anomalie de résistance). La modélisation d'un fusible est donc assez compliquée, c'est pourquoi nous avons préféré établir une loi approchée du phénomène, et cela à partir de nos constatations expérimentales. Un fusible est constitué par une simple feuille de cuivre très fine $(0,02 \mathrm{~mm})$ serrée très fortement entre plusieurs couches de tissu de verre. Nous avons mesuré, à chaque essai, le courant et la tension à ses bornes, ce qui nous a permis de calculer les variations temporelles de sa résistance et de l'énergie absorbée. Ensuite par élimination du temps, il a été facile de construire un graphique du type :

$$
\frac{R_{\mathrm{F}}}{R_{\mathrm{F}_{\mathrm{o}}}}=f\left(\frac{W}{M}\right)
$$

où est tracé, en fonction de l'énergie absorbée par unité de masse, le rapport entre les résistances du fusible à chaud et à froid.

Les courbes $R_{\mathrm{F}} / R_{\mathrm{F}_{0}}=f(W / M)$ dépendent beaucoup de la densité de courant traversant le fusible [20]. Cependant, lors des essais effectués, la densité de courant maximale n'a pas beaucoup varié. En outre, quand le temps de latence du fusible passait de 15 à $30 \mu \mathrm{s}$, l'énergie absorbée pour multiplier par $120 \mathrm{sa}$ résistance changeait seulement de 2,7 à $2,9 \mathrm{~kJ} / \mathrm{g}$. Nous avons donc pu, après un certain nombre d'essais, prendre une courbe moyenne et considérer qu'elle restait valable pour toutes les expériences. Cette courbe moyenne est tracée figure 11, et il suffit ainsi de calculer, à chaque pas de temps, l'énergie absorbée par le fusible

$$
W\left(t^{\prime}\right)=\int_{0}^{t^{\prime}} R_{\mathrm{F}} I_{\mathrm{F}}^{2} \mathrm{~d} t
$$

pour en déduire la nouvelle valeur de $\boldsymbol{R}_{\mathrm{F}}$. 


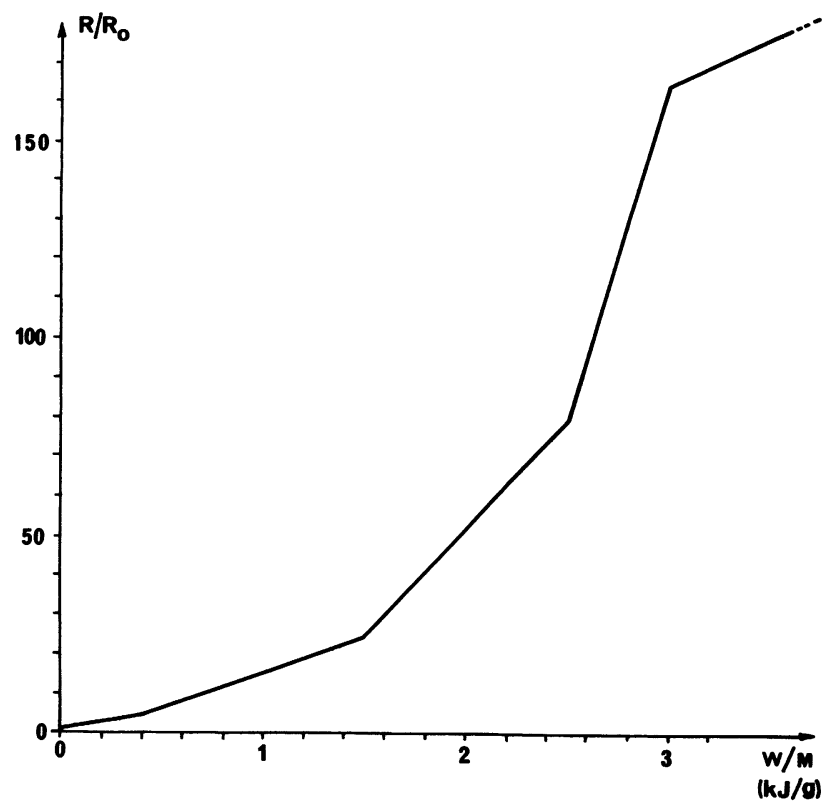

Fig. 11. - Variation de la résistance d'un fusible en cuivre en fonction de l'énergie absorbée (la densité de courant est comprise entre 0,5 et $1 \times 10^{5} \mathrm{~A} / \mathrm{mm}^{2}$ ).

[Variation of a copper fuse resistance as a function of its absorbed energy (the maximum current density is included between 0.5 and $\left.1 \times 10^{5} \mathrm{~A} / \mathrm{mm}^{2}\right)$.]

2.3.4 Procédure de calcul. - Là encore, nous avons employé une méthode numérique pas à pas. A l'instant initial $t_{\mathrm{i}}$ l'arc est traversé par un courant $I_{\mathrm{i}}$ et, à ses bornes, nous avons une tension $V\left(t_{i}\right)$. A partir de ces valeurs on déduit $g\left(t_{\mathrm{i}}\right)=I_{\mathrm{i}} / V\left(t_{\mathrm{i}}\right)$, $\tau\left(I_{\mathrm{i}}\right)$ et $G\left(I_{\mathrm{i}}\right)$, si bien que l'équation (17) permet de trouver la nouvelle conductance $g\left(t_{\mathrm{i}}+\Delta t\right)$. Ensuite, par résolution des équations électriques du système (eqs. (11 à 15) incluses) et de celles donnant $R_{\mathrm{F}}(20)$ et (21) nous déduisons la nouvelle valeur du courant d'arc $I\left(t_{\mathrm{i}}+\Delta t\right)$, et donc aussi des fonctions $G$ et $\tau$ relatives à ce nouveau courant. Après, nous calculons la tension d'arc $V\left(t_{\mathrm{i}}+\Delta t\right)=I\left(t_{\mathrm{i}}+\Delta t\right) / g\left(t_{\mathrm{i}}+\Delta t\right)$ qui servira de base pour le calcul de l'échelon suivant.

Pour que les erreurs dues à la quasi-linéarisation de l'arc ne faussent pas le calcul, il faut choisir un pas de temps $\Delta t$ qui soit petit relativement à $\tau$, par exemple $\Delta t=\tau / 50$. L'expression du courant à chaque instant nous donne celle de la force $F_{\mathrm{e}}$ et par suite nous permet de calculer la loi du mouvement par :

$$
2 \bar{\omega} d e \frac{\mathrm{d} v}{\mathrm{~d} t}=F_{\mathrm{e}} .
$$

2.4 Modélisation de La QUatrième PHASE. - La quatrième et dernière phase de fonctionnement du disjoncteur à couteaux commence à la fin du transfert de courant de l'ouvreur vers le fusible et correspond à la période de réisolement, pendant laquelle le courant dans l'ouvreur est très faible, en même temps que le fusible génère progressivement une forte tension transitoire aux bornes de l'ouvreur.
2.4.1 Hypothèses. - Il est évident que la théorie de l'arc précédente reste valable et qu'a priori les équations électriques de la troisième phase devraient être aussi utilisées maintenant. C'est d'ailleurs ce que nous avons fait pour commencer, avec malheureusement des résultats décevants. En effet la modélisation de l'arc traversé par des courants proches de zéro est déjà intrinsèquement délicate et, dans notre cas particulier, la situation est encore plus compliquée puisque le courant d'arc est le résultat de l'interaction des deux courants $I_{0}$ et $I_{\mathrm{C}}$ avec le circuit extérieur. L'utilisation des équations électriques de la troisième phase dans la modélisation du réisolement du disjoncteur se traduisait alors par des instabilités numériques, avec des oscillations autour de zéro qui augmentaient progressivement d'amplitude.

Les difficultés que nous venons d'énumérer nous ont conduits à simplifier notre modèle, de façon à échapper aux instabilités numériques, en gardant toutefois la théorie de l'arc présentée auparavant. La seule simplification introduite a été de négliger l'influence du courant de commande $I_{\mathrm{C}}$ dans le réisolement, ce qui a priori ne doit pas être grossièrement faux. En effet, la tension appliquée à l'ouvreur par le fusible qui chauffe est plusieurs fois supérieure à la tension résiduelle aux bornes de commande, sauf au tout début de cette quatrième phase. Le fait de négliger le circuit de commande dans l'étude du réisolement ne doit donc pas donner des résultats très éloignés de la réalité. Tel a bien été le cas, comme nous allons le montrer maintenant.

2.4.2 Equations. - Pendant cette dernière phase, les tensions $V_{\mathrm{A} 1}$ et $V_{\mathrm{A} 2}$ fournies par les arcs respectivement traversés par les courants $\frac{1}{2}\left(I_{C}+I_{0}\right)$ et $\frac{1}{2}\left(I_{C}-I_{0}\right)$ pendant la troisième phase, sont toujours modélisés à partir de (17) mais il est important de remarquer que, bien que maintenant $I_{\mathrm{C}}$ soit supposé nul, $V_{\mathrm{A} 1}$ et $V_{\mathrm{A} 2}$ ne sont pas égales car le "passé » des conductances qui les gouvernent est différent.

En remarquant que l'inductance $L_{0 \mathrm{P}}$ de l'ouvreur est très faible comparée à celle du circuit principal $L_{\mathbf{P}}$ de même que $R_{0} I_{0}$ vis-à-vis des tensions d'arcs $V_{\mathrm{A} 1}$ et $V_{\mathrm{A} 2}$, les équations (11), (12) et (13) se réduisent à :

$$
\begin{aligned}
\frac{\mathrm{d} I_{0}}{\mathrm{~d} t}=\frac{1}{L_{\mathrm{P}}}\left(V_{\mathrm{P}}-R_{\mathrm{P}}\left(I_{0}+\right.\right. & \left.\left.I_{\mathrm{F}}\right)-V_{\mathrm{A} 1}-V_{\mathrm{A} 2}\right)- \\
& \quad-\frac{1}{L_{\mathrm{F}}}\left(V_{\mathrm{A} 1}+V_{\mathrm{A} 2}-R_{\mathrm{F}} I_{\mathrm{F}}\right) \\
\frac{\mathrm{d} I_{\mathrm{F}}}{\mathrm{d} t} & =\frac{1}{L_{\mathrm{F}}}\left(V_{\mathrm{A} 1}+V_{\mathrm{A} 2}-R_{\mathrm{F}} I_{\mathrm{F}}\right) \\
I_{\mathrm{C}} & =0
\end{aligned}
$$

celle gouvernant $V_{\mathrm{P}}(15)$ restant inchangée.

Enfin, les courants étant très faibles, les efforts sur les conducteurs sont négligeables et :

$$
\frac{\mathrm{d} v}{\mathrm{~d} t}=0 \text {. }
$$




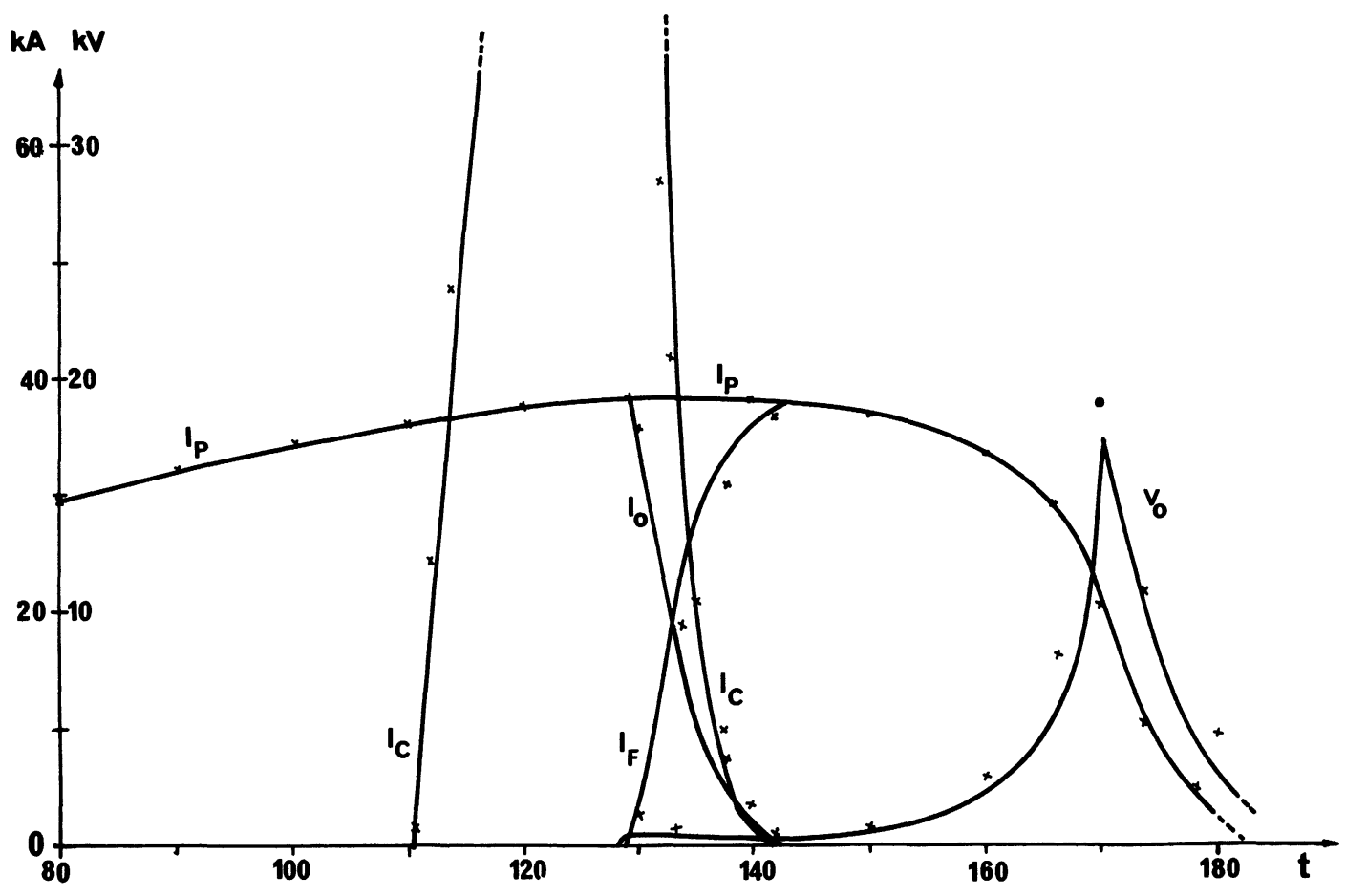

Fig. 12. - Modélisation d'une expérience. La courbe est théorique, les points sont expérimentaux. Les courants et tension sont définis figure 6 .

[Simulation of a typical experiment. The curve is theoretical, while points are experimental. Currents and voltage were defined on figure 6.]

2.5 Résultats NUMÉRIQUes. - La figure 12 montre la simulation théorique et les résultats d'une expérience dans laquelle les tensions de charge des bancs de condensateur étaient :

$$
V_{\mathrm{PO}}=2030 \mathrm{~V}, \quad V_{\mathrm{C} 0}=4000 \mathrm{~V} \text {. }
$$

L'impulsion de commande est envoyée $110 \mu$ s après le début de l'impulsion principale et le nombre de couteaux en face de chaque conducteur est de 8 . Nous constatons que la simulation est tout à fait correcte.

Sur la figure 13 sont indiqués le déplacement $x$ et la vitesse $v$ des morceaux conducteurs situés entre 2 couteaux consécutifs, lors de la simulation théorique d'un essai typique. L'apparition des arcs de rupture, qui correspond à un déplacement $\Delta x$ égal à $2 \mathrm{~mm}$ (épaisseur du conducteur plus épaisseur d'un couteau), doit avoir lieu selon le calcul vers $18,5 \mu \mathrm{s}$, ce qui est tout à fait'conforme aux expériences.

La figure 14 donne la courbe théorique de réisolement intrinsèque (pas de couteaux de réisolement). Pour la tracer, nous avons simulé plusieurs essais, en changeant à chaque fois les dimensions du fusible et la tension de charge du banc de condensateurs principal. Puis, nous avons divisé la tension théorique de reclaquage dans l'ouvreur par le nombre de couteaux. Cette courbe a été tracée pour des essais où l'amplitude du courant de commande restait voisine de $165 \mathrm{kA}$ et où la pression était de 50 bars; elle est donc à comparer avec la courbe notée 0 de la figure 5 . La similitude entre les deux courbes est frappante, ce qui montre que la modélisation post-arc de l'ouvreur est en gros correcte. A partir de $18 \mu \mathrm{s}$, la courbe théorique dépasse la courbe expérimentale. Ceci est normal, car notre modélisation ne tient compte que des reclaquages thermiques et non pas des reclaquages diélectriques, qui doivent devenir prépondérants à partir de $20 \mu$ s.

3. Conclusion. - Nous avons présenté un interrupteur ultra-rapide à couteaux, dont la commande est du type par pression magnétique directe. Celle-ci s'est révélée très favorable du point de vue énergétique : nous pouvons escompter des rapports energie de commande/énergie principale de l'ordre de $10 \%$ pour des temps d'ouverture de $6 \mu \mathrm{s}$. La quantité de matière détruite à chaque fonctionnement est très faible. Ce disjoncteur est tout à fait adapté à la réalisation d'une source d'énergie rapide à partir d'un stockage inductif. Nous avons d'autre part modélisé le fonctionnement de ce disjoncteur, ce qui a nécessité en particulier de déterminer certaines constantes capables de décrire les arcs dans les conditions de nos expériences (électrodes d'aluminium, pression d'azote de 50 bars) avec des formules simples classiques. Ceci nous a permis de retrouver avec une très bonne précision la courbe de réisolement expérimentale. 


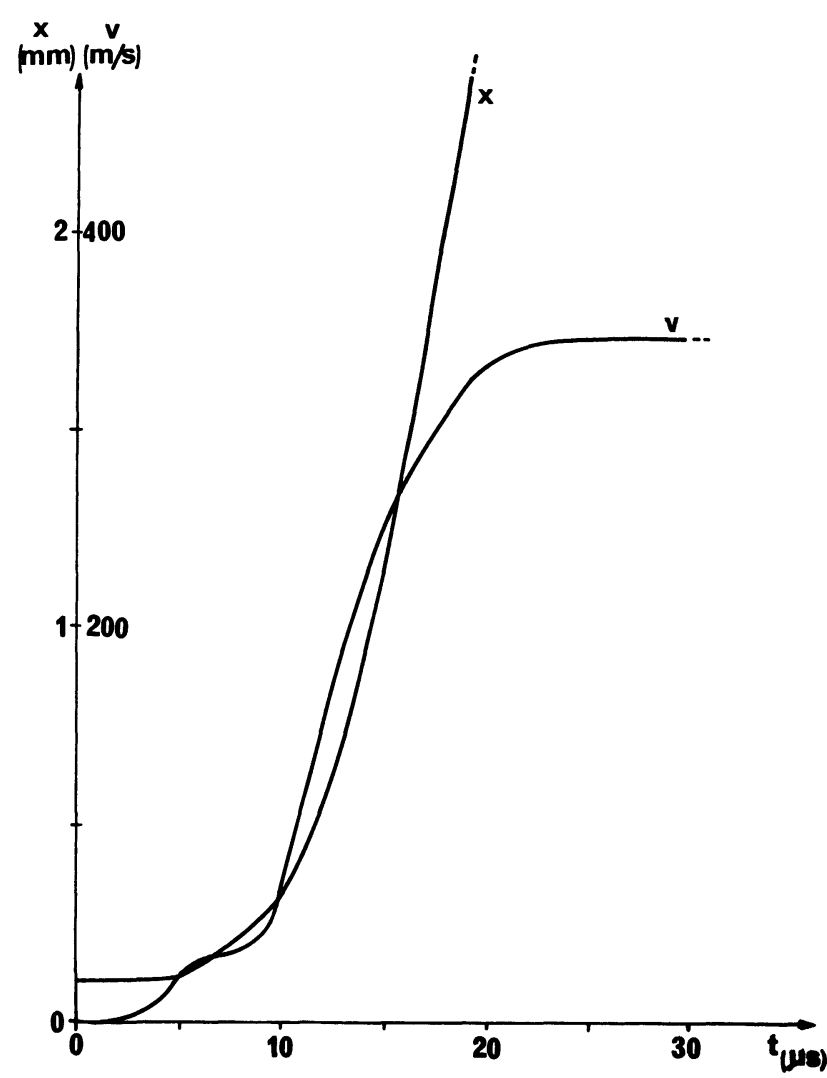

Fig. 13. - Vitesse des conducteurs et distance parcourue en fonction du temps écoulé depuis le début de l'impulsion de commande, d'après les résultats du calcul.

[Theoretical speed and displacement of the conductors as a function of the time spent from the start of the command current.]

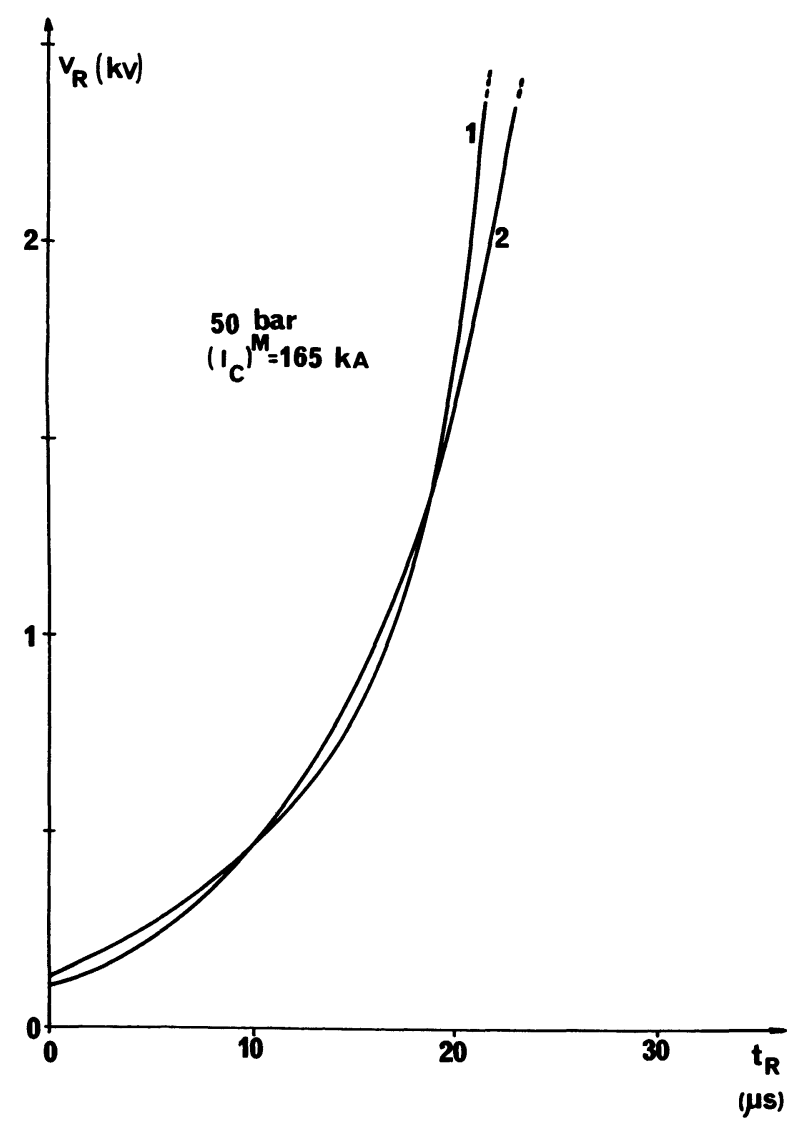

Fig. 14. - Courbes de réisolement intrinsèque : (1) est théorique, (2) est expérimentale.

[Intrinsic reinsulation curves : (1) is theoretical, (2) is experimental.]

\section{Bibliographie}

[1] Sultanem, F., Bleiss, C. A., Rioux, C., RiouxDamidau, F., J. Appl. Phys. 52 (1981) 3655.

[2] Rioux-Damidau, F., Congrès Electrotechnique Mondial, Moscou, juin 1977, communication $\mathrm{n}^{\circ}$ 2-64.

[3] ForD, R. D., VitKovitsky, I. M., NRL Memorandum Report 3561 (1977).

[4] Leroux, B., Rioux-Damidau, F., Revue Phys. Appl. 14 (1979) 705.

[5] Caupers, P., Rioux, C., Rioux-Damidau, F., Rev. Sci. Instrum. 52 (1981) 81.

[6] Pelenc, Y., Merlin-Gerin Technical News 2 (1976) f1.

[7] Urbanek, J., Proc. IEEE 59 (1971) no 4.

[8] Caupers, P., Thèse de Docteur-ingénieur, Université Paris-VI, 1980.

[9] Hahn, R., Thèse d'Etat, Université Paris-VI, 1975.

[10] CAssie, A. M., Rapport CIGRE 102, 1939.

[11] MAYR, O., Electrotech.Zeit.64(1943)645/652.
[12] Brown, T. E., A.I.E.E. Trans. on Pas. 77 (1959) 1508, 1516.

[13] RIEDER, W., Rapport CIGRE 107 (1966).

[14] Hochrainer, A., GrÜTZ, A., Rapport CIGRE (13-12) 1972.

[15] Heroin, P., Séminaire sur les arcs électriques (EDF), Milly-la-Forêt, 1973.

[16] Ayrton, H., The Electrician Series (D. Van Nostrand Company, New York) 1902.

[17] Techniques de l'ingénieur D 305-4.

[18] TuCKer, T. J., J. Appl. Phys. 32 (1961) 1894.

[19] Dimarco, J., Burckhardt, L., J. Appl. Phys. (1970) 3894.

[20] Antoni, B., Revue Phys. Appl. 9 (1974).

[21] Benford, J., Cal vin, H., Conf. on En. storage, Compr. and Switching Venise 1974, pages 39/50. 